\title{
Dermatology
}

\section{Cardiovascular Disease and Risk Factors among Psoriasis Patients in Two US Healthcare Databases, 2001-2002}

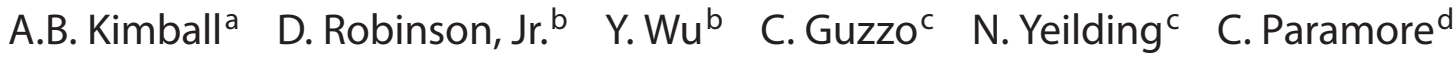 \\ K. Fraeman ${ }^{d}$ M. Balab

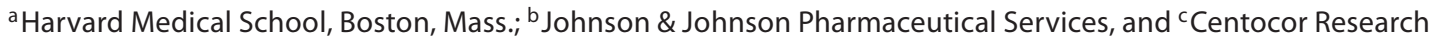 \\ and Development, Centocor, Inc., Malvern, Pa.; ${ }^{\mathrm{d}}$ United BioSource Corp (UBC), Bethesda, Md., USA
}

\section{Key Words}

Psoriasis • Dermatology • Comorbidity • Treatment • Epidemiology $\cdot$ Autoimmune

\begin{abstract}
Background: Cardiovascular diseases or risk factors (CVDR) seem to be more common in psoriasis patients than in the general population. Objective: We assessed the relationship of psoriasis with CVDR by analysis of healthcare claims data using a cross-sectional, prevalence-based study design. $\mathbf{P a}$ tients and Methods: The IMS Health and MarketScan ${ }^{\circledR}$ Claims databases were used to identify adults with psoriasis diagnostic codes. Non-psoriasis controls were matched 3:1 based on age, gender, census region and previous medical insurance coverage. Odds ratios evaluated the relative prevalence of CVDR, and Mantel-Haenszel confidence intervals were estimated. Results: CVDR prevalence was generally higher in psoriasis patients than controls in both datasets. Odds ratios for atherosclerosis, congestive heart failure, type 2 diabetes, and peripheral vascular disease were $\geq 1.20$ for psoriasis patients. Elevated disease severity was associated with a higher rate of CVDR, but varied somewhat by dataset and condition. Conclusions: Elevated CVDR rates were found in psoriasis patients compared with controls. This pattern merits further examination.

Copyright $\odot 2008$ S. Karger AG, Basel
\end{abstract}

\section{Introduction}

Several studies have demonstrated that cardiovascular diseases and their associated risk factors are more common in patients with psoriasis than in the general population [1-3]. The cause of this elevated risk is unclear. Some authors suggest that the profound psychological impact of psoriasis may drive risky behaviors such as obesity and smoking, and thereby directly increase cardiovascular risk [4]. Others have suggested that there may be some intrinsic associated risk: elevated lipids have been documented in psoriasis patients at the time of their initial diagnosis when compared to non-psoriasis controls that were matched for BMI status, as well as other demographic, clinical, and lifestyle characteristics [5].

New evidence has led to the hypothesis that psoriasis is a systemic inflammatory state that confers increased cardiovascular risk above and beyond traditional risk factors. Several cytokines that have been identified as important mediators of psoriasis, such as interleukins-1, 4, 6, 8, 12 and tumor necrosis factor- $\alpha$, have also been identified in metabolic syndrome, a chronic inflammatory state associated with obesity [6-12]. Further supporting this concept is the work of Prodanowich et al. [13], demonstrating that treatment of psoriasis patients with methotrexate (MTX) substantially decreases the risk of cardiovascular events, especially when supplemented with folate.

\section{KARGER}

Fax +4161306 1234 E-Mail karger@karger.ch www.karger.com
Don Robinson, Jr, MSPH

Johnson \& Johnson Pharmaceutical Services

200 Great Valley Parkway

Malvern, PA 19355 (USA)

Tel. +1 610240 8468, Fax +1 610651 6717, E-Mail drobins4@cntus.jnj.com 
Table 1. ICD-9 codes for identifying cardiovascular diseases and risk factors

\begin{tabular}{ll}
\hline Condition & ICD-9 code \\
\hline Cerebrovascular disease (CVD) & $430 . \mathrm{x}-438 . \mathrm{x}$ \\
Congestive heart failure (CHF) & $428 . \mathrm{x}$ \\
Diabetes mellitus, type 2(DMT2) & $250 . \mathrm{x} 0,250 . \mathrm{x} 2$ \\
Hyperlipidemia & $272.0-4$ \\
Hypertension (HTN) & $401 . \mathrm{x}$ \\
Ischemic heart disease (IHD) & $410 . \mathrm{x}-414 . \mathrm{x}$ \\
Acute myocardial infarction (AMI) & $410 . \mathrm{x}$ \\
Peripheral vascular disease (PVD) & $440 . \mathrm{x}, 441 . \mathrm{x}, 443 . \mathrm{x}, 447.1,557.1,557.9, \mathrm{~V} 43.4$ \\
$\quad$ Atherosclerosis & $440 . \mathrm{x}$ \\
\hline
\end{tabular}

ICD-9 = International Classification of Diseases, 9th revision.

One limitation of the early epidemiologic studies examining cardiac risk in psoriasis patients is that they generally sampled hospitalized or specialty clinic patients, and were therefore likely biased toward patient populations with more severe disease $[1,14,15]$. Since most psoriasis patients have mild disease, generalizing from the inpatient population may overestimate cardiovascular risk. This limitation is important because several recent studies using healthcare databases have demonstrated that higher disease severity is associated with higher cardiovascular risk, as has been demonstrated in the arthritis population $[3,16,17]$. Data from a large dataset of ambulatory patients from the United Kingdom (UK), the General Practice Resource Database (GPRD), confirmed the elevated prevalence of cardiovascular risk factors and acute myocardial infarction in a general population of psoriasis patients. Consistent with other recent healthcare database studies, they found that the risk was increased in those who were receiving systemic therapy, a marker they used as a surrogate for disease severity [11, $18]$.

This study was designed to examine the risk of cardiovascular diseases and their related risk factors in the US population by using two large health plan datasets containing medical, as well as prescription claim data. Obesity is more common in the US than in the UK [19], and patterns of medical care, as well as treatment, differ across the countries. This in turn may lead to differences in the relationships between psoriasis treatment, disease severity, and cardiac risk. Using these databases, we were able to examine the medications prescribed for the treatment of cardiovascular diseases or risk factors (CVDR), some of which have implications for the management of psoriasis and its comorbidities.

\section{Patients and Methods}

A cross-sectional, prevalence-based study design was used for this investigation. Adult patients, defined as at least 17 years of age in 2001, were selected from two US healthcare claims databases: the IMS Health Integrated Claims Database (IMS Health) and the MarketScan ${ }^{\circledR}$ Commercial Claims and Encounters Database (MarketScan). Patients had to be continuously enrolled with medical and drug benefits from January 1, 2001 to December 31, 2002 , but an enrollment gap of up to 60 days was allowed. Patients with psoriasis were identified as having at least 1 medical service claim at any time between January 1, 2001 and December 31, 2002, with an International Classification of Diseases, 9th Revision (ICD-9) code of 696.0 or 696.1 within any diagnostic field. Psoriatic arthritis was considered a clinical subtype of psoriasis. Patients without psoriasis, who also required at least 1 medical service claim during the 2 -year study period, were randomly selected as controls from the initial study population and frequency matched at a 3:1 ratio to each psoriasis patient based on age $( \pm 2$ years), gender (exact), region (exact), and length of prebaseline medical insurance coverage (exact to the month). Matching on the duration of prebaseline medical insurance ensured a similar likelihood for chronic conditions between cases and controls [21]. No psoriasis ICD-9 codes, as defined above, were allowed in the treatment record of the control group.

Two datasets were used for validation purposes, where findings in one were considered robust if confirmed in the other. The initial MarketScan population was about 6 million patients during the observation period (2001-2002). The datasets had some representation of the elderly through a few retiree and Medicare health plans that offered medical and drug coverage. During this time period, most MarketScan patients had healthcare benefits provided through self-insured employers, many being Fortune 500 firms. About 11 million patients were originally available for study with IMS Health, where healthcare coverage was provided by mostly commercial plans of various types. Both datasets were mainly midwestern and southern in their regional characteristics and have been often used for epidemiology and cost of illness studies. Additional details on these data sources have been previously published [20]. 
Cardiovascular conditions and their risk factors were selected based on the public health importance of these comorbidities [3, $11,13,16,18]$. Cardiovascular diseases and risk factors were identified through ICD-9 codes commonly used in epidemiologic literature (table 1). To ensure maximum sensitivity to CVDR comorbidities, only 1 medical service claim with the appropriate ICD-9 code was required within any diagnostic field. All CVDR events that occurred at any point over the 2-year observation period were captured for analysis. Documentation of CVDR drug therapies was based on National Drug Code (NDC) identifiers found on the prescribed drug claims as well as on Healthcare Common Procedure Coding System (HCPCS) codes. As with diagnostic data, a prescription drug claim that occurred at any point in the 2-year observation period was included in pharmacotherapy analysis.

To explore disease severity patterns, patients were grouped into 2 levels: Mild and Severe. Severe patients received at least 1 systemic treatment during the time period while everyone else was classified as Mild (table 2) [11, 16]. CVDR rates were compared between the psoriasis and matched control cohorts by using the odds ratio (OR) statistic. The Mantel-Haenszel method was used to estimate the $95 \%$ confidence interval (CI) for the OR between the psoriasis and matched control population [22]. A single programmer who was experienced with both datasets wrote a common code for data table construction and conducted quality assurance on the data reported. A second programmer confirmed the coding logic and consistency. The study was exempt from Institutional Review Board (IRB) processes because the patient data in both datasets were de-identified and fully compliant with the Health Insurance Portability and Accountability Act (HIPAA). Version 8.2 of the SAS system (SAS Institute, Cary, N.C., USA) was used to generate all statistical analyses.

Several sensitivity analyses were conducted. One sensitivity analysis raised the psoriasis case identification criteria from all patients with at least 1 medical service claim to all patients with at least 1 inpatient or at least 2 ambulatory face-to-face encounters $[21,23,24]$. New controls were generated for this analysis using the same matching criteria as used for the main population model. A second sensitivity analysis compared CVDR rates using different utilization thresholds within the nonsystemic and systemic psoriasis treatment categories. This sensitivity analysis used patients from the main population model, but varied the analytical approach. Based on the observed distribution of utilization data, 2 psoriasis treatment thresholds were chosen for this analysis: 3 or more claims and 8 or more claims, when contrasting CVDR rates with controls.

\section{Results}

\section{Demographics, Prevalence and Treatment Patterns}

Psoriasis and control patients were very similar in demographic profile as a result of the matching program, with no difference in age and gender. Some variability occurred in regional representation, particularly with MarketScan. Psoriasis patients in both datasets had demographic profiles consistent with the literature from
Table 2. Psoriasis medical and drug treatments by type

\begin{tabular}{ll}
\hline Classification & Treatment \\
\hline Nonsystemic & Laser treatment \\
& Topical \\
& UVA/UVB \\
\hline Systemic & Antimetabolites \\
& Biologics \\
Cyclosporine \\
Hydroxyurea \\
Leflunomide \\
Methotrexate (MTX) \\
Mycophenolate \\
Photochemotherapy, unspecified \\
PUVA \\
Retinoid \\
Sulfasalazine
\end{tabular}

Antimetabolites = azathioprine, mercaptopurine and thioguanine; biologics = between 2001 and 2002, etanercept (Enbrel) or infliximab (Remicade); laser treatment = CPT 96920-96922; photochemotherapy, unspecified $=$ Goeckerman regimen and $/$ or PUVA (CPT 96913); PUVA = combination therapy involving psoralen cream and ultraviolet A phototherapy (PUVA; CPT 96912); topical = anthralin, calcipotriene, coal tar, corticosteroid cream, salicylic acid, tacrolimus cream, tazarotene; UVA/UVB = ultraviolet A or B phototherapy (CPT 96900 and 96910).

multi-site observational studies on ambulatory psoriasis patients, although they tended to be older, depending on the dataset [25-28]. Patients in IMS Health were younger than those from MarketScan (table 3), and in both datasets the patients with psoriasis were older and more likely to be male than in the overall population from which they were chosen [20]. The prevalence of psoriasis was similar across the datasets: $0.79 \%$ in MarketScan and $0.89 \%$ in IMS Health (table 3 ).

Treatment patterns were consistent across the datasets, with a few exceptions. Between 18 and $27 \%$ of psoriasis patients, depending on the dataset, had no recorded use of any of the specified psoriasis treatments over the 2-year observation period. Between 59 and $67 \%$ of patients received only nonsystemic treatment (table 4). Psoriasis patients having a history of only nonsystemic treatment $(65 \%[\mathrm{n}=13,394]$ and $57 \%[\mathrm{n}=14,578]$ of all psoriasis patients in MarketScan and IMS Health, respectively) were almost always using topical monotherapy (data not shown). Topical treatment was combined with ultraviolet (UV) treatment in $2-3 \%$ of patients across the datasets (data not shown). Among patients having a record for both systemic and nonsystemic treat- 
Fig. 1. Psoriasis CVDR risk compared to controls. Odds ratios (OR) and confidence intervals (CI) based on a unique patient count of those having 1 or more medical claims within the period. CVD $=$ Cerebrovascular disease; $\mathrm{CHF}=$ congestive heart failure; DMT2 = type 2 diabetes mellitus; HTN = hypertension; IHD = ischemic heart disease; AMI = acute myocardial infarction; PVD = peripheral vascular disease.

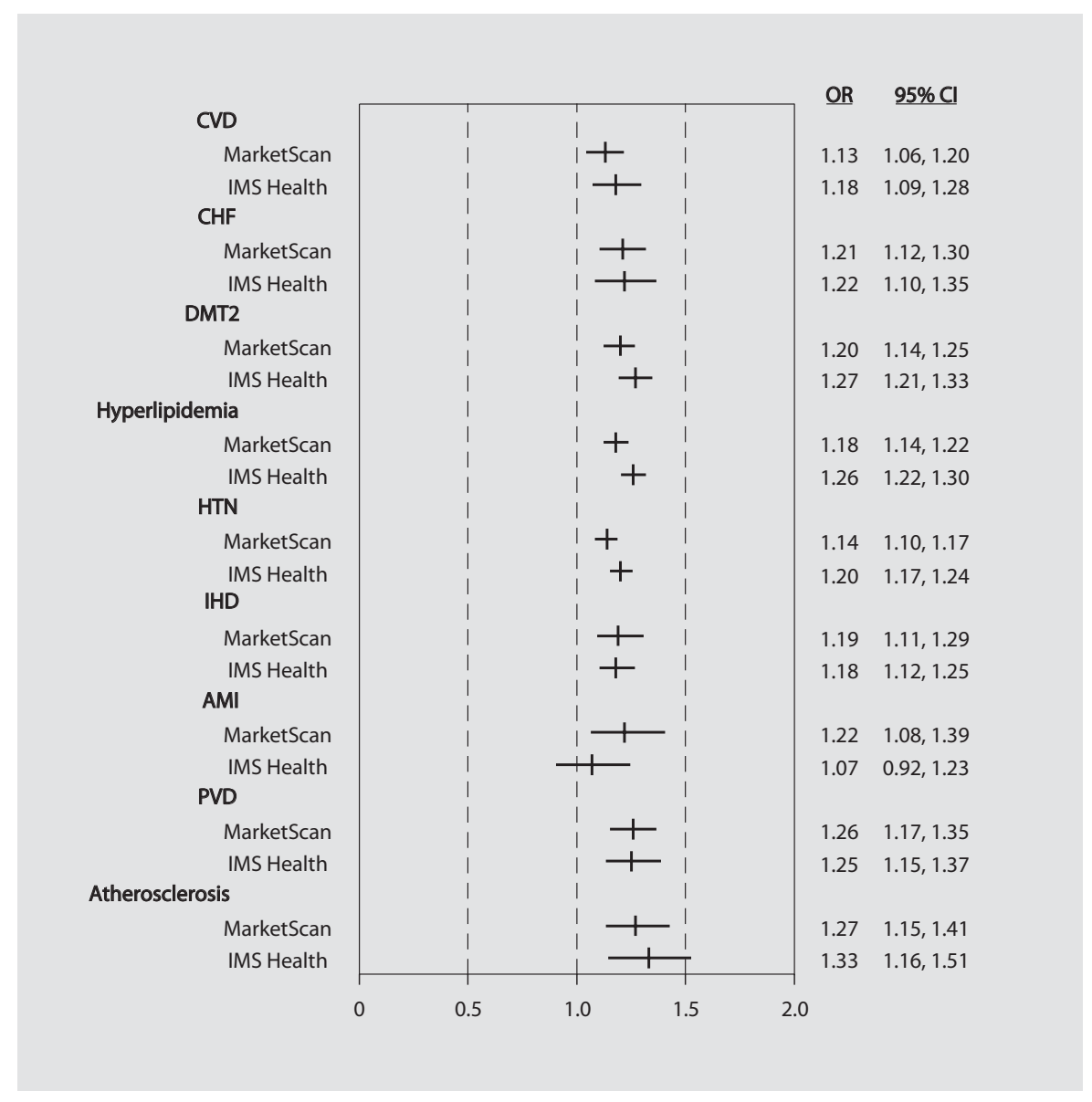

Table 3. Control and psoriasis population characteristics ${ }^{1}$

\begin{tabular}{|c|c|c|c|c|c|}
\hline \multirow{2}{*}{\multicolumn{2}{|c|}{ Population }} & \multicolumn{2}{|l|}{ MarketScan } & \multicolumn{2}{|l|}{ IMS Health } \\
\hline & & control & psoriasis & control & psoriasis \\
\hline \multirow{3}{*}{\multicolumn{2}{|c|}{$\begin{array}{l}\text { Prevalence }^{2}, \mathrm{n}(\%) \\
\text { Age }^{3}, \text { mean } \pm \text { SD (median) } \\
\text { Female, n }(\%)\end{array}$}} & $82,456(\mathrm{~N} / \mathrm{A})$ & $20,614(0.79)$ & $101,507(\mathrm{~N} / \mathrm{A})$ & $25,556(0.89)$ \\
\hline & & $54.9 \pm 15.5(55)$ & $54.9 \pm 15.5(55)$ & $47.1 \pm 12.7(48)$ & $47.1 \pm 12.6(48)$ \\
\hline & & $43,416(52.7)$ & $10,854(52.7)$ & $52,010(51.2)$ & $13,096(51.2)$ \\
\hline \multirow[t]{4}{*}{ Census region ${ }^{3}, \%$} & $\mathrm{E}$ & 18.5 & 22.6 & 27.8 & 27.8 \\
\hline & MW & 32.2 & 33.9 & 42.0 & 41.9 \\
\hline & $\mathrm{S}$ & 42.1 & 37.5 & 23.9 & 24.0 \\
\hline & W & 7.0 & 5.9 & 6.3 & 6.3 \\
\hline
\end{tabular}

$\mathrm{N} / \mathrm{A}=$ Not applicable; $\mathrm{E}$ = east, $\mathrm{MW}=$ midwest, $\mathrm{S}$ = south, $\mathrm{W}=$ west.

${ }^{1}$ Psoriasis and cases defined as $\geq 1$ medical service claim without a medical service claim for ICD-9 696.0 or 696.1 (control) and $\geq 1$ medical service claim with ICD-9 696.0 or 696.1 (psoriasis).

2 Two-year prevalence based on a population of 2,619,719 adults continuously enrolled from 1/1/2001 through 12/31/2002 for medical benefits in MarketScan and of 2,872,333 in IMS Health [20].

${ }^{3}$ Based on 2001 data. US Census Region percentages may not equal 100 due to rounding and missing data. 
Table 4. Psoriasis treatment utilization ${ }^{1}$

\begin{tabular}{|c|c|c|c|c|c|}
\hline \multirow[t]{2}{*}{ Treatment category $^{2}$} & \multirow[t]{2}{*}{ Treatment type } & \multicolumn{2}{|c|}{ MarketScan } & \multicolumn{2}{|c|}{ IMS Health } \\
\hline & & $\mathrm{n}$ & $\%$ & $\mathrm{n}$ & $\%$ \\
\hline No treatment ${ }^{3}$ & & 3,689 & 17.9 & 6,931 & 27.1 \\
\hline \multirow[t]{5}{*}{ Nonsystemic treatment } & Laser & 0 & 0.0 & 1 & 0.0 \\
\hline & Topical & 16,094 & 78.1 & 17,371 & 68.0 \\
\hline & UVA/UVB & 748 & 3.6 & 932 & 3.6 \\
\hline & Nonsystemic combinations & 392 & 1.9 & 446 & 1.8 \\
\hline & All nonsystemic & 13,843 & 67.2 & 15,159 & 59.3 \\
\hline Less than systemic & None or only nonsystemic (mild) & 17,532 & 85.0 & 22,090 & 86.4 \\
\hline \multirow{14}{*}{ Systemic treatment } & Antimetabolites & 69 & 0.3 & 101 & 0.4 \\
\hline & Biologics & 381 & 1.8 & 384 & 1.5 \\
\hline & Cyclosporine & 160 & 0.8 & 224 & 0.9 \\
\hline & Hydroxyurea & 37 & 0.2 & 29 & 0.1 \\
\hline & Leflunomide & 195 & 0.9 & 240 & 0.9 \\
\hline & MTX & 1,563 & 7.6 & 1,817 & 7.1 \\
\hline & Mycophenolate & 33 & 0.2 & 43 & 0.2 \\
\hline & Photochemotherapy, unspecified & 10 & 0.0 & 5 & 0.0 \\
\hline & PUVA & 645 & 3.1 & 710 & 2.8 \\
\hline & Retinoids & 511 & 2.5 & 529 & 2.1 \\
\hline & Sulfasalazine & 437 & 2.1 & 516 & 2.0 \\
\hline & Nonsystemic \& systemic combinations & 2,866 & 13.9 & 3,112 & 12.2 \\
\hline & Systemic only & 216 & 1.0 & 354 & 1.4 \\
\hline & All systemic (severe) & 3,082 & 15.0 & 3,466 & 13.6 \\
\hline
\end{tabular}

UVA/UVB = Ultraviolet A or B phototherapy; MTX = methotrexate; PUVA = combination therapy involving psoralen cream and ultraviolet A phototherapy.

${ }^{1}$ Results from all psoriasis patients with $\geq 1$ medical service claim with ICD-9 696.0 or 696.1. Denominator is based on the number of psoriasis patients having $\geq 1$ medical claim over the 2-year period. MarketScan: $\mathrm{n}=$ 20,614; IMS Health: $n=25,556$. Percentages may not sum to 100 due to rounding.

2 Prescribed drug categories based on Red Book (Thomson Healthcare) definitions. 'All nonsystemic' includes any nonsystemic treatment but excludes any systemic treatment; 'all systemic' includes any systemic treatment and allows nonsystemic treatments in the patient history.

${ }^{3}$ Patients without a medical or prescribed drug claim for any psoriasis treatment, as defined.

Table 5. Cardiovascular disease and risk factor events by population and dataset ${ }^{1}$

\begin{tabular}{|c|c|c|c|c|}
\hline \multirow[t]{2}{*}{ Condition } & \multicolumn{2}{|l|}{ MarketScan } & \multicolumn{2}{|l|}{ IMS Health } \\
\hline & $\begin{array}{l}\text { psoriasis } \\
(\mathrm{n}=20,614)\end{array}$ & $\begin{array}{l}\text { control } \\
(\mathrm{n}=82,456)\end{array}$ & $\begin{array}{l}\text { psoriasis } \\
(\mathrm{n}=25,556)\end{array}$ & $\begin{array}{l}\text { control } \\
(\mathrm{n}=101,507)\end{array}$ \\
\hline Cerebrovascular disease & $1,344(6.5)$ & $4,812(5.8)$ & $784(3.1)$ & $2,655(2.6)$ \\
\hline Congestive heart failure & $873(4.2)$ & $2,917(3.5)$ & $496(1.9)$ & $1,617(1.6)$ \\
\hline Diabetes mellitus, type 2 & $2,750(13.3)$ & $9,398(11.3)$ & $2,605(10.2)$ & $8,336(8.2)$ \\
\hline Hyperlipidemia & $6,432(31.2)$ & $22,941(27.8)$ & $8,065(31.6)$ & $27,239(26.8)$ \\
\hline Hypertension & $7,308(35.5)$ & $26,886(32.6)$ & $7,497(29.3)$ & $26,037(25.7)$ \\
\hline Ischemic heart disease & $944(4.6)$ & $3,185(3.9)$ & $1,987(7.8)$ & $6,746(6.6)$ \\
\hline Acute myocardial infarction & $323(1.6)$ & $1,060(1.3)$ & $240(0.9)$ & $895(0.9)$ \\
\hline Peripheral vascular disease & $1,014(4.9)$ & $3,260(4.0)$ & $691(2.7)$ & $2,200(2.2)$ \\
\hline Atherosclerosis & $498(2.4)$ & $1,576(1.9)$ & $301(1.2)$ & $904(0.9)$ \\
\hline
\end{tabular}

${ }^{1}$ Reporting patient counts and prevalence (\%) for studied cardiovascular disease or risk factor (CVDR conditions. 


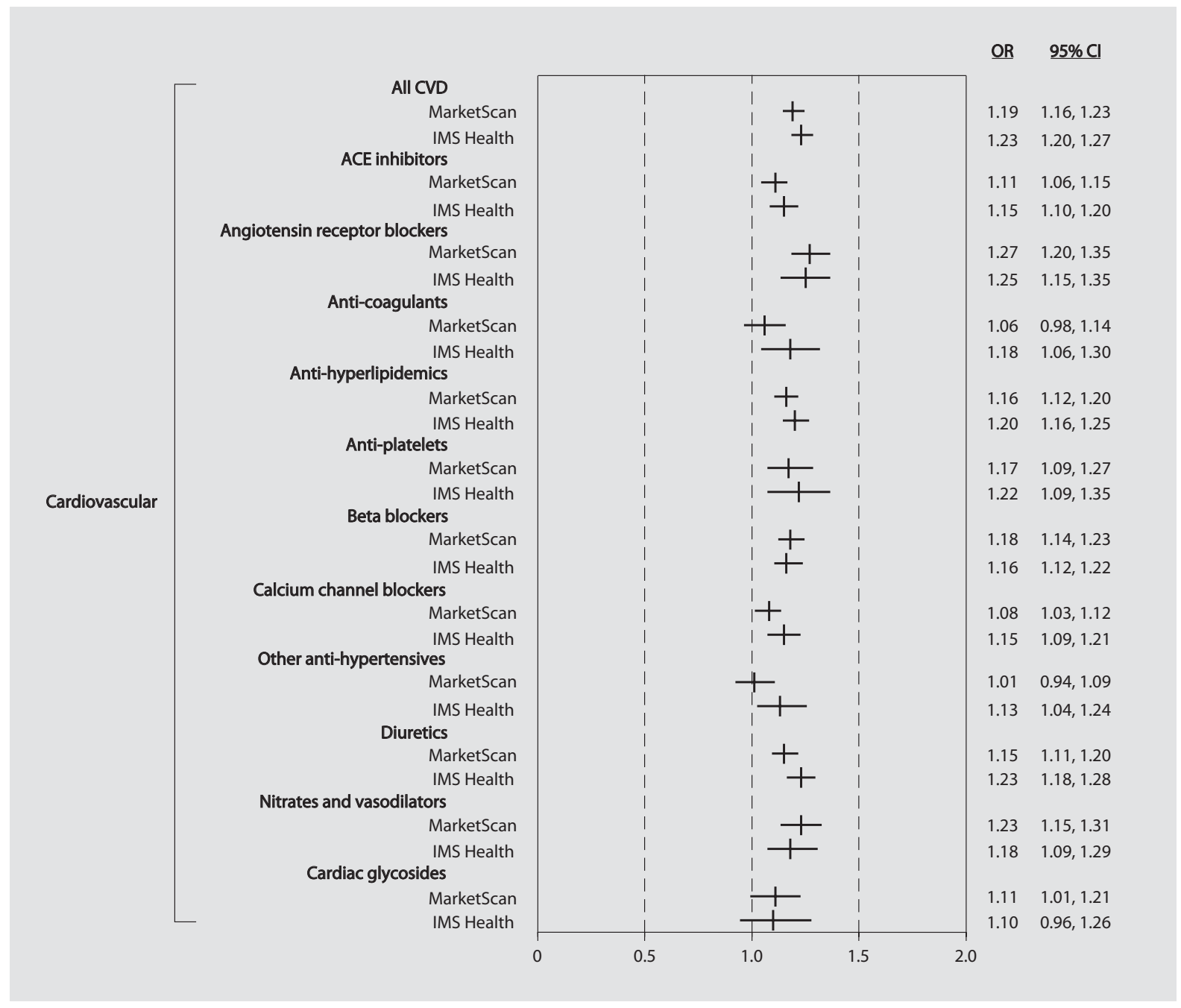

Fig. 2. Psoriasis cardiovascular drug utilization compared to controls. Odds ratios (OR) and confidence intervals (CI) based on a unique patient count of those having 1 or more drug claims within the period. Prescribed drug categories are based on Red Book (Thomson Healthcare) definitions. Anticoagulants include warfarin and heparin; antihyperlipidemics include statins, fibrates, resins and niacin. Diuretics include multiple diuretic combination products (e.g., ACE inhibitors or angiotensin receptor blockers).

ments, the top 3 combinations were consistent across the data sources: topical/MTX (3\%), topical/psoralen ultraviolet A (PUVA) (1\%) and topical/retinoid (1\%; data not shown). A severe form of psoriasis was found in approximately 15 and $14 \%$ of patients in the MarketScan and IMS Health databases, respectively, when use of systemic treatment was the marker for psoriasis severity (table 5).

\section{Cardiovascular Diseases and Risk Factors}

A greater prevalence of CVDR was observed in the psoriasis group in both datasets when compared with controls (table 5; fig. 1). The ORs were similar across MarketScan and IMS Health, with the lower confidence limit generally $>1.00$. The highest ORs consistently meeting or exceeding 1.20 were for atherosclerosis, congestive heart failure (CHF), type 2 diabetes mellitus, and peripheral vascular disease (PVD).

Medications typically used to manage CVDR were also used more frequently among psoriasis patients than controls (fig. 2, 3). The magnitude of difference between the psoriasis and control groups in the drug-based analysis was similar to that found when using ICD-9-based medical claims. CVDR risk generally increased with dis- 


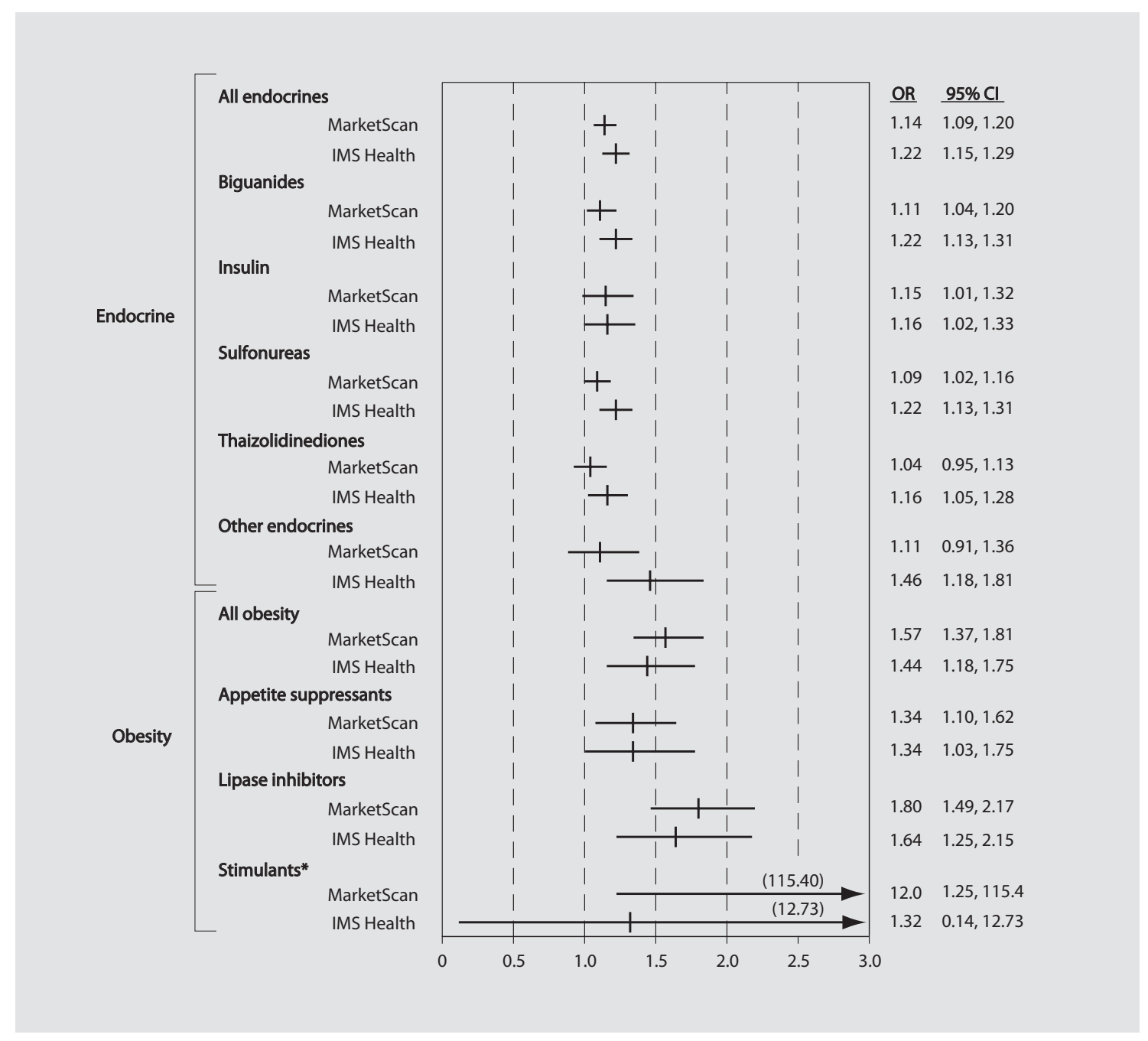

Fig. 3. Psoriasis endocrine and obesity drug utilization compared to controls. Odds ratios (OR) and confidence intervals (CI) based on a unique patient count of those having 1 or more drug claims within the period. Prescribed drug categories are based on Red Book (Thomson Healthcare) definitions. Appetite suppressants include benfluorex, diethylptopion, mazindol, phendimetrazine, phentermine and sibutramine. Other endocrines include $\alpha$-glucosidase inhibitors, glucogon and meglitinides. * Stimulant use was very low across both datasets.

ease severity, using psoriasis drug utilization as the indicator for severity (fig. 4, 5). In both datasets, higher disease severity raised the risk for CHF, type 2 diabetes mellitus, hypertension, and ischemic heart disease (fig. 4).

The case-selection sensitivity analysis (at least 1 inpatient or at least 2 ambulatory face-to-face encounters) produced similar CVDR patterns, although with lower ORs. In this analysis, a higher prevalence for atherosclerosis and $\mathrm{CHF}$ was found in patients with psoriasis than in controls in IMS Health (OR $=1.23$ for both). However, atherosclerosis and CHF had CI lower limits that ap- proached 1.00, while some other CVDR exceeded 1.00. The treatment utilization threshold analysis (3 or more, 8 or more) produced a stepped pattern of increasing CVDR risk that was similar to that found with the severity analysis (data not shown).

\section{Discussion}

Consistent with previous research, this study found greater occurrence of CVDR among psoriasis patients when using data from 2 large US claims databases. While 
Fig. 4. Psoriasis cardiovascular diseases and risk factors by severity level. Odds ratios (OR) and confidence intervals (CI) based on a unique patient count of those having 1 or more drug claims within the period. Based on psoriasis treatment intensity, where Mild patients have no treatment or only nonsystemic treatment and Severe patients have at least 1 systemic treatment, with nonsystemic treatment allowed. Abbreviations as in figure 1. ${ }^{*} \mathrm{High}$ er risk by disease severity in both datasets, with the lower CI limit $>1.00$.

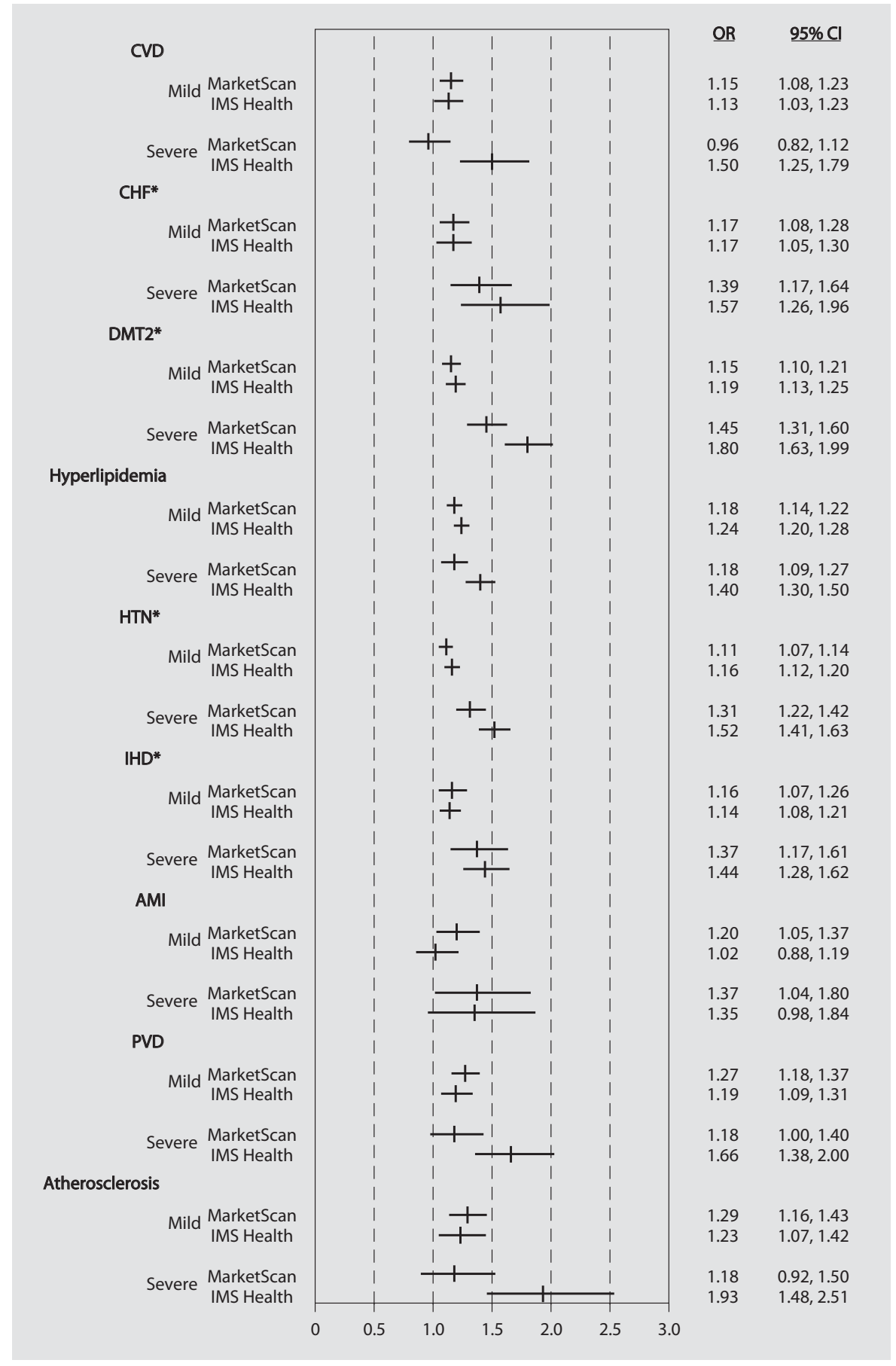

the increased CVDR occurrence in psoriasis patients is modest for any given disease or condition, the pattern is pervasive. The prevalence of CVDR rose with disease severity when using standard psoriasis treatments as surrogates for psoriasis severity. Other studies have found these relationships [29], but this is the largest investiga- tion of CVDR prevalence among psoriasis patients that used US population-level datasets. The consistency of the CVDR patterns across the datasets is notable, as these information sources represent several million US patients from multiple regions of the country who were covered by a variety of health insurance plans. In a related paper 
Fig. 5. CVDR therapies by psoriasis severity level. Odds ratios (OR) and confidence intervals (CI) based on a unique patient count of those having 1 or more drug claims within the period. Based on psoriasis treatment intensity, where Mild patients have no treatment or at least 1 nonsystemic treatment and no history of systemic treatment. Severe patients have at least 1 systemic treatment, with nonsystemic treatment allowed.

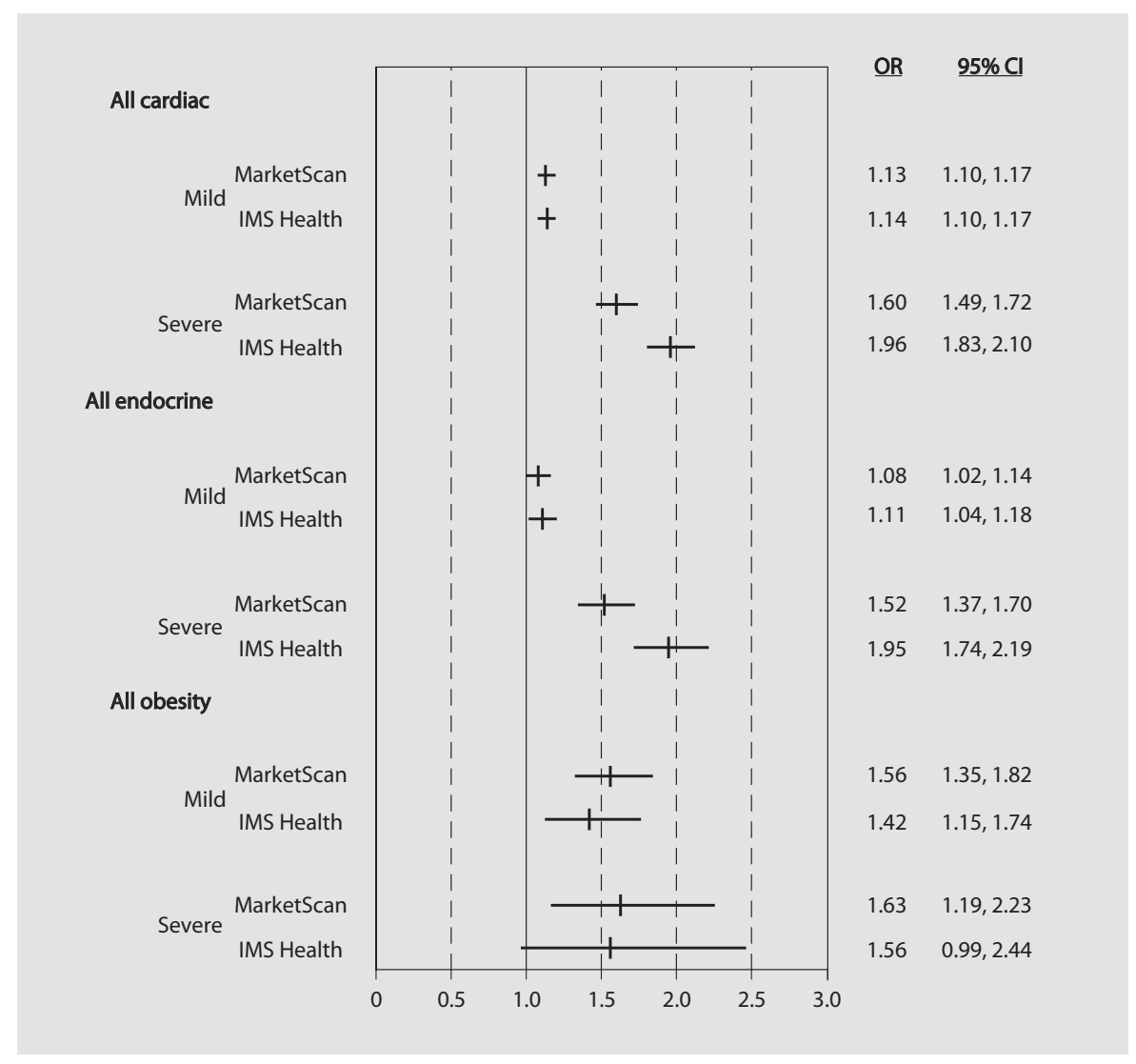

using IMS Health data, Han et al. [17] found higher rates for CVDR among psoriatic arthritis patients except for hyperlipidemia than reported here among all psoriasis patients.

Our method for identifying patients with psoriasis, cardiovascular diseases and their associated risk factors depended on the reliability of medical claims coding and administrative processing within real-world treatment environments. Psoriasis prevalence rates in these datasets $(0.79-0.89 \%)$ were fairly close to the lower range that appears in the literature (1-2\%), although they were higher than the $0.5 \%$ found among Swedish military conscripts and lower than the $1.5 \%$ reported in the UK $[8,12$, 30-32]. The minor difference in prevalence across the datasets may result from the observed disparity in age and variation in coding practices, as well as differences in health benefits, particularly regarding access to dermatologists. Nonsystemic treatments were the main therapies for these patients across both datasets; the use of systemic treatment was quite low. The most frequent treatment pattern was the use of multiple nonsystemic treatments. When systemic treatment was administered to a patient it was almost always accompanied by nonsystemic treatment.

A number of limitations to these data need to be noted. A cross-sectional, prevalence-based study design was used in this investigation, which combines new and old cases. Consequently, the impact of disease duration in our analysis is unclear and causality cannot be established. Healthcare claim datasets lack information on many important patient attributes, such as height, weight, ethnicity, health behaviors (smoking, exercise, alcohol consumption, diet) and family medical history, all of which may function as unknown confounders in the analysis. No measure of disease severity was available. Laboratory data in both datasets were too infrequent to be reliably incorporated into this study. A healthy worker effect is quite likely in this population because it was comprised of stable employees and their family members.

These results also reflect US population treatment patterns at a particular point in time, and confirmation of 
these findings in US data sources with stronger Western representation, as well as Medicaid patients, would be useful. Due to the proprietary source of the datasets, verification of ICD-9 coding by medical chart review could not be conducted. However, quality assurance tests are regularly conducted upon these data by the suppliers. The advice from several external experts with experience in using US healthcare claim datasets for psoriasis research (see Acknowledgements) suggests that there is a good true positive case identification rate when using psoriasis ICD-9 diagnostic codes, although the false negative rate among the control population is unknown. The 2-year observation period used in this study has been found to raise the sensitivity of case identification in a Medicare population for several other chronic conditions when using claims-based diagnostic codes [24]. The increased precision of higher utilization thresholds for claimsbased case identification needs to be balanced with selection effects since in our datasets, raising the utilization thresholds for case identification notably lowered the prevalence rate to a point well below published reports (data not shown).

In spite of their limitations, healthcare claims data have been widely used for epidemiologic research in psoriasis as well as other autoimmune diseases because they are readily available and contain useful clinical information drawn from large population samples $[3,11,13,16-$ $18,23,31]$. The risk of an observation bias in this study seems small because the CVDR patterns reported here were also found, albeit muted, among the mild psoriasis patients. Mild psoriasis patients had medical utilization only slightly higher than controls. Similarly, these CVDR patterns continued when the medical utilization of the psoriasis controls was raised in the sensitivity analysis. Psoriasis and controls had the same level of medical utilization in the sensitivity analysis (data not shown).

It is difficult to untangle the complex relationships between disease severity, treatments, and patient characteristics in retrospective claims database studies because there is, appropriately, a relationship between these factors in daily medical practice. Prospective, longitudinal studies are needed to further explore pathogenic linkages between psoriasis and cardiovascular disease [29, 33]. Our knowledge of psoriasis pathogenesis, patient health, and treatment effectiveness would be greatly enriched if biomarkers, clinical assessments, and patient quality-oflife questionnaires were incorporated into such studies $[4,5,32]$. The results from this study may have implications for the clinical care of psoriasis patients if they are confirmed by prospective, longitudinal studies. If elevat- ed CVDR risk is related to psoriasis pathogenesis, more aggressive management of cardiovascular disease and related risk factors may be warranted, especially for the more severely afflicted patients.

\section{Acknowledgements}

The authors appreciate the research insight provided by Steve Feldman, MD, PhD, of Wake Forest University; Harold Javitz, $\mathrm{PhD}$, of SRI International; Robert Kirsner, $\mathrm{MD}$, PhD, of the University of Miami; Adam Long, PhD, of Gordian Health Solutions, and Dave Margolis, MD, PhD, of the University of Pennsylvania. The data support provided by Debra Eisenberg, PhD, Ning Zhao, $\mathrm{PhD}$, and Stephen $\mathrm{Xu}, \mathrm{MS}$, of Centocor was indispensable, as was the editorial and submission support provided by Kristin Ruley Sharples, PhD, Rebecca E. Clemente, PhD, Mary Ann Thomas, RN, BSN, and Mary Whitman, PhD, of Centocor. We gratefully recognize the clinical advice of Catherine Cabot, $\mathrm{MD}$, and Larry Deckelbaum, MD, of Centocor, regarding cardiovascular pharmacotherapy.

\section{Conflict of Interest}

Funding for this study was provided by Centocor, Inc., Malvern, Pa. Drs. Bala, Guzzo, Yeilding, Wu, and Mr. Robinson were employees of Centocor, Inc. at the time of manuscript preparation. Dr. Kimball has served as a consultant, investigator, a paid speaker for Centocor, and has a fellowship funded by Centocor. Mr. Paramore and Ms. Fraeman have served as research consultants and analysts for Centocor.

References $D_{1}$ Henseler T, Christophers E: Disease concomitance in psoriasis. J Am Acad Dematol 1995;32:982-986

2 Poikolainen K, Karvonen J, Pukkala E: Excess mortality related to alcohol and smoking among hospital-treated patients with psoriasis. Arch Dermatol 1999;135:14901493.

3 Mallbris L, Akre O, Granath F, et al: Increased risk for cardiovascular mortality in psoriasis inpatients but not in outpatients. Eur J Epidemiol 2004;19:225-230.

-4 Kimball AB, Jacobson C, Weiss S, et al: The psychosocial burden of psoriasis. Am J Clin Dermatol 2005;6:383-392.

5 Mallbris L, Granath F, Hamsten A, Ståhle M: Psoriasis is associated with lipid abnormalities at the onset of skin disease. J Am Acad Dermatol 2006;54:614-621.

6 Krueger JG: The immunologic basis for the treatment of psoriasis with new biologic agents. J Am Acad Dermatol 2002;46:1-23; quiz 23-26. 
7 Ghoreschi K, Thomas P, Breit S, et al: Interleukin- 4 therapy of psoriasis induces Th2 responses and improves human autoimmune disease. Nat Med 2003;9:40-46.

8 Lebwohl M: Psoriasis. Lancet 2003;361: 1197-1204.

-9 Mallbris L, Ritchlin CT, Stahle M: Metabolic disorders in patients with psoriasis and psoriatic arthritis. Curr Rheumatol Rep 2006; 8:355-363.

10 Najarian DJ, Gottlieb AB: Connections between psoriasis and Crohn's disease. J Am Acad Dermatol 2003;48:805-821; quiz 822824.

-11 Neimann AL, Shin DB, Wang X, et al: Prevalence of cardiovascular risk factors in patients with psoriasis. J Am Acad Dermatol 2006;55:829-835.

-12 Schön MP, Boehncke WH: Psoriasis. N Engl J Med 2005;352:1899-1912.

13 Prodanowich S, Ma F, Taylor JR, et al: Methotrexate reduces incidence of vascular diseases in veterans with psoriasis or rheumatoid arthritis. J Am Acad Dermatol 2005;52: 262-267.

14 McDonald CJ, Calabresi P: Psoriasis and occlusive vascular disease. Br J Dermatol 1978; 99:469-475.

- 15 Ena P, Madeddu P, Glorioso N, et al: High prevalence of cardiovascular diseases and enhanced activity of the renin-angiotensin system in psoriatic patients. Acta Cardiol 1985;40:199-205.

-16 Crown WH, Bresnahan BW, Orsini LS, et al: The burden of illness associated with psoriasis: cost of treatment with systemic therapy and phototherapy in the US. Curr Med Res Opin 2004;20:1929-1936.
17 Han C, Robinson DW Jr, Hackett MV, et al: Cardiovascular disease and risk factors in patients with rheumatoid arthritis, psoriatic arthritis, and ankylosing spondylitis. J Rheumatol 2006;33:2167-2172.

18 Gelfand JM, Neimann AL, Shin DB, et al: Risk of myocardial infarction in patients with psoriasis. JAMA 2006;296:1735-1741.

19 Banks J, Marmot M, Oldfield Z, Smith JP: Disease and disadvantage in the United States and in England. JAMA 2006;295: 2037-2045.

20 Robinson D Jr, Hackett M, Wong J, et al; the IMID Study Group: Co-occurrence and comorbidities in patients with immune-mediated inflammatory disorders: an exploration using US healthcare claims data, 2001-2002. Curr Med Res Opin 2006;22:989-1000.

21 Peterson E, Van Vorst K, Wickstrom S, et al: Exploring issues in managed care: Six illustrative case studies; in McGlynn EA (ed): The Effect of Chronic Disease on Risk of Disenrollment from Five United HealthcareAffiliated Health Plans. RAND, 2001, vol 11, pp 16-20.

22 Woodward M: Epidemiology: Study Design and Data Analysis, ed 1. Boca Raton, Chapman \& Hall, 1999, pp 113-117.

23 Feldman SR, Evans C, Russell MW: Systemic treatment for moderate to severe psoriasis: estimates of failure rates and direct medical costs in a north-eastern US managed care plan. J Dermatolog Treat 2005;16:37-42.

24 Rector TS, Wickstrom SL, Shah M, et al: Specificity and sensitivity of claims-based algorithms for identifying members of Medicare+Choice health plans that have chronic medical conditions. Health Serv Res 2004;39:1839-1857.
25 Lundberg L, Johannesson M, Silverdahl M, et al: Health-related quality of life in patients with psoriasis and atopic dermatitis measured with SF-36, DLQI and a subjective measure of disease activity. Acta Derm Venereol 2000;80:430-434.

26 National Institute of Arthritis and Musculoskeletal and Skin Diseases: Health Information. Available from http://www.niams. nih.gov/hi/topics/psoriasis/psoriasis.htm [accessed 2006 Aug 18].

27 Mallbris L, Larsson P, Bergqvist S, et al: Psoriasis phenotype at disease onset: clinical characterization of 400 adult cases. J Invest Dermatol 2005;124:499-504.

-28 Uttjek M, Dufåker M, Nygren L, Stenberg B: Determinants of quality of life in a psoriasis population in northern Sweden. Acta Derm Venereol 2004;84:37-43.

-29 Kremers HM, McEvoy MT, Dann FJ, Gabriel SE: Heart disease in psoriasis. J Am Acad Dermatol 2007;57:347-354.

30 Landgren E, Bråbäck L, Hedlin G, et al: Psoriasis in Swedish conscripts: time trend and association with T-helper 2-mediated disorders. Br J Dermatol 2006;154:332-336.

$>31$ Gelfand JM, Weinstein R, Porter SB, et al: Prevalence and treatment of psoriasis in the United Kingdom: a population-based study. Arch Dermatol 2005;141:1537-1541.

32 Langley RGB, Krueger GG, Griffiths CEM: Psoriasis: epidemiology, clinical features, and quality of life. Ann Rheum Dis 2005; 64(suppl 2):ii18-ii23.

33 Wakkee M, Thio HB, Prens EP, et al: Unfavorable cardiovascular risk profiles in untreated and treated psoriasis patients. Atherosclerosis 2007;190:1-9. 